difference in the enzyme activity between the untreated and stimulated cells in all treatment groups. However, a dose dependent decrease was observed only in case of CD14 cells. Both unsorted monocytes and monocyte sub-populations showed a significant decrease in the concentration of cortisol measured when co-incubated with carbenoxolone, indicative of the direct involvement of $11 \beta$-HSD1 enzyme in the conversion of cortisone to cortisol.

Conclusions: The results of this study showed that IL-17 induced GC insensitivity might be dependent on the reduced $11 \beta$-HSD1 enzyme activity in inflammatory conditions. We showed that the pro-inflammatory cytokine IL-17 causes a significant decrease in the $11 \beta$-HSD1 enzyme activity.

Disclosure of Interest: None declared

DOI: 10.1136/annrheumdis-2017-eular.2681

\section{AB0046 METABOLISM AND OSTEOARTHRITIS ARE LINKED BY ADIPOKINES}

M.-L. Hülser ${ }^{1}$, C. Schreiyäck ${ }^{1}$, Y. Luo ${ }^{2}$, A. Bozec ${ }^{2}$, G. Schett ${ }^{2}$, E. Neumann ${ }^{1}$, U. Müller-Ladner ${ }^{1} .{ }^{1}$ Internal Medicine and Rheumatology,

Justus-Liebig-University Giessen, Bad Nauheim; ${ }^{2}$ Clinic of Medicine 3 -

Immunology and Rheumatology, University of Erlangen-Nuremberg, Erlangen, Germany

Background: Obesity and hyperinsulinemia are of increasing importance in the Western society. Both obesity and insulin resistance lead to changes in expression of adipokines such as adiponectin, visfatin or leptin, which appear to be immunomodulatory factors also in rheumatic diseases.

Objectives: Since osteoarthritis (OA) is often accompanied by hyperinsulinemia and obesity, we combined both mouse models (destabilization of the medial meniscus (DMM) and high-fat diet (HFD)). Here, we evaluated and correlated the systemic and local effects of both models at different states of OA development with special focus on the local/systemic expression of the adipokines adiponectin, visfatin and leptin over time.

Methods: HFD (mainly consisting of saturated fatty acids) to induce obesity and hyperinsulinemia, and ND (normal diet) as control were fed to C57BI/6 mice for 3 months followed by surgical OA induction (time point 0 ). Tissues and sera were collected at different time points after DMM-mediated OA induction (4, 6, 8 weeks). Adipocytokine (leptin, visfatin, adiponectin and IL-6) serum levels were measured by ELISA. Histological stainings of the joints $(H / E$, safranin $O$, pappenheim and Masson-Goldner's trichrome) were evaluated and arthritis progress was scored. Immunohistochemical stainings of the joints were performed to evaluate the local distribution of adipokines, which were correlated to systemic adipocytokine levels and the respective arthritis score.

Results: Low systemic IL-6 levels confirmed that no acute inflammation due to surgery or infection was present in all animals. OA induction was visible at all time points, which was aggravated in HFD compared to ND mice (OA score: 4 weeks ND 0.87 vs. HFD $0.93,6$ weeks ND 1.44 vs. HFD $3.69,8$ weeks ND 1.78 vs. HFD 2.18). Systemic levels of leptin were significantly induced by HFD confirming the induction of insulin resistance, but DMM decreased leptin levels at all time points (significantly for 3 out of 6 groups, e.g. 4 weeks: HFD healthy vs. HFD DMM 18.4 $\mathrm{ng} / \mathrm{ml}$ vs. $3.7 \mathrm{ng} / \mathrm{ml}$ ). Interestingly, the systemic increase of adiponectin by DMM was time dependent (only 8 weeks after surgery: HFD healthy vs. HFD DMM 5176 $\mathrm{ng} / \mathrm{ml}$ vs. $6149 \mathrm{ng} / \mathrm{ml}$ ) but independent of diet. However, HFD in combination with DMM did not show significant effects on systemic levels of adiponectin, visfatin or IL-6.

Conclusions: HFD deteriorates OA in the DMM model. Systemic leptin levels were elevated by HFD/insulin resistance but reduced by DMM, which could not be observed for the mainly proinflammatory adipokine visfatin. Of note, systemic inflammation as shown by systemic IL- 6 levels was low in all animals. The stage of $\mathrm{OA}$ development influences adiponectin levels, which were only increased systemically 8 weeks after surgery. In summary, systemic levels of adipokines are altered by DMM and HFD as well as the combination of both models and the analyzed adipokines show differing reactions to these factors.

Disclosure of Interest: None declared

DOI: 10.1136/annrheumdis-2017-eular.4913

\section{AB0047 TYPE I INTERFERON IS HIGHLY EXPRESSED IN RA SYNOVIAL FLUID AND JOINT CARTILAGE CORRELATED WITH SERUM RHEUMATOID FACTOR; A PRELIMINARY EXPERIMENTAL STUDY}

M. Nakayama ${ }^{1}$, H. Tobimatsu ${ }^{1}$, H. Imamura ${ }^{1}$, Y. Sakuma ${ }^{1}$, K. Yano ${ }^{1}$, Y. Niki ${ }^{2}$, K. Ikari ${ }^{1} .{ }^{1}$ Department of Orthopedic Surgery, Institute of Rheumatology, Tokyo Women's Medical University; ${ }^{2}$ Department of Orthopedic Surgery, Keio University, Tokyo, Japan

Background: It was reported that type I interferon (IFN) is involved in the pathogenesis of rheumatoid arthritis (RA) [1-3], while the relevance of the IFN signature to RA disease activity and progression remains unclear. There were few reports about the expression of IFN in synovial fluid and joint cartilage.

Objectives: The aim of this study is to investigate the role of IFN in the pathogenesis of RA by means of the analysis of joint tissues of RA patients comparing with those of osteoarthritis $(\mathrm{OA})$ patients.

Methods: Synovial fluid, synovia and cartilage were collected from RA and
OA patients ( $\mathrm{n}=10$ for each) during total knee arthroplasty in our hospital and blood samples were collected just before surgery. As preoperative therapy for RA, Methotrexate (MTX) was administered to 9 patients (dose ranged (4-12mg), DMARDS without MTX to 2, biological DMARDS to 2, Prednisolone (PSL) to 6 (dose $1-5 \mathrm{mg}$ ). Quantities of IFN alpha or beta of blood and joint fluid were measured with ELISA (PBL Assay Science, USA), and expression of IFN alpha, beta and TNF alpha of synovia and joint cartilage were measured with real time PCR. In RA patients. Serum biomarkers such as C-reactive protein (CRP), erythrocyte sedimentation rate (ESR), Rheumatoid Factor (RF), hemoglobin and platelet were measured and investigated correlation with the quantities of IFN of blood or joint tissues. Medication for RA were investigated as well.

Results: In blood and synovial fluid of RA patients, IFN alpha and beta were highly detected, while they were not detected in those of OA patients. The expression of IFN alpha and beta of RA cartilage were much higher than those of OA whereas they were not expressed in synovium of both RA and OA. Expression of IFN was not correlated with that of TNF alpha in RA patients. Statistical analysis revealed that RF was related with blood and joint IFN and other markers were not. Medications for RA were not correlated with IFN expression.

Conclusions: IFN was highly expressed in RA synovial fluid, joint cartilage and blood, not in OA. IFN immunotherapy has been reported to induce RA [4-5], therefore abundant IFN might induce RA and inhibit cure of RA. Our results showed that RF was related with blood and joint IFN. It can be speculated that $\mathrm{RF}$ might be an index of IFN regulation in RA patient, however, more samples must be investigated to prove this speculation.

References:

[1] de Padiila CML and Niewold TB. The Type I Interferons: Basic Concepts and Clinical Relevance in Immune-mediated Inflammatory Diseases. Gene. 2016; $15,576,14-21$

[2] Carrio JR, de Paz B, et al. IFN alpha Serum Levels Are Associated with Endothelial Progenitor Cells Imbalance and Disease Features in Rheumatoid Arthritis Patients. Plos One, 2014;9, e86069.

[3] van Holten J, Smeets TJM, et al. Expression of interferon b in synovial tissue from patients with rheumatoid arthritis: comparison with patients with osteoarthritis and reactive arthritis. Ann Rheum Dis. 2005; 64: 1780-2.

[4] Passos SE, Evangelista SPT, et al. Rheumatoid arthritis induced by alphainterferon therapy. Clin Rheumatol. 2001;20(4):297-9.

[5] Cacopardo B, Benanti F, et al. Rheumatoid arthritis following PEG-interferonalfa-2a plus ribavirin treatment for chronic hepatitis $\mathrm{C}$ : a case report and review of the literature. BMC research notes. 2013; 6:437.

Disclosure of Interest: None declared

DOI: 10.1136/annrheumdis-2017-eular.4978

\section{AB0048 ANTIPHOSPHOLIPID ANTIBODIES, INTERLEUKIN-6 AND TUMOR NECROSIS FACTOR- $\alpha$ IN ATHEROSCLEROTIC PROCESS IN PATIENTS WITH RHEUMATOID ARTHRITIS AND SYSTEMIC LUPUS ERYTHEMATOSUS}

N.A. Bashlakova ${ }^{1}$, T.D. Tyabut ${ }^{2}$, A.E. Buglova ${ }^{2} .{ }^{1}$ Ultrasound Diagnostics Department; ${ }^{2}$ Cardiology and Rheumatology Department, Belarusian Medical Academy of Postgraduate Education, Minsk, Belarus

Background: Systemic inflammation has been postulated to be an independent cardiovascular risk factor, particularly in patients with autoimmune rheumatic disorders (ARD), such as rheumatoid arthritis (RA) and systemic lupus erythematosus (SLE), and is associated with accelerated atherosclerosis. There is some evidence to suggest that antiphospholipid antibodies (aPL) may also play a role in the development of atherosclerosis. However, it is few data about the relationship between these autoantibodies and inflammatory mediators in the development of atherosclerosis

Objectives: To clarify the involvement of inflammatory mediators and aPL in the atherosclerotic process in patients with ARD.

Methods: The study included 87 female patients with ARD (RA ( $n=47)$, mean age $45,0(33,0 ; 51,0)$ years old, disease duration $9,0(3,0 ; 14,0)$ years, disease activity (DAS28 $=5,37(4,69 ; 5,86)$ points); SLE $(n=40)$, mean age $33,5(27,5 ; 44,5)$ years old, disease duration $8,0(5,0 ; 14,5)$ years, disease activity SLEDAI-2K 7,0 (4,0; $11,5)$ points). Sixty healthy women (mean age $40,5(36,0 ; 47,0)$ years old) formed the control group.

The levels of high sensitive C-reactive protein (hs-CRP), interleukin-6 (IL-6), tumor necrosis factor - $\alpha$ (TNF- $\alpha)$, LA, IgG/lgM antibodies to cardiolipin (aCL), 32-glucoprotein-1(aß2-GP1), annexin $\mathrm{V}(\mathrm{aAnV})$ and prothrombin (aPT)) were determined with ELISA. Intima-media thickness (IMT) of the carotid artery wall and the presence of atherosclerotic plaques were revealed ultrasonographically according to the described ESH/ESC Guidelines.

Results: The levels of hs-CRP, IL-6, TNF- $\alpha$ were significantly higher in ARD patients than in the control group, which indicates the disease activity. Furthermore, the patients with SLE had a significant correlation between IL- 6 and SLEDAI-2K $(r=0,471, p=0,002)$, TNF $-\alpha$ and SLEDAI-2K $(r=0,499, p=0,001)$, whereas the patients with RA had only significant correlation between hs-CRP and DAS28 $(r=0,355, p=0,031)$.

The concentration of $\lg \mathrm{aCL}$, IgG and $\lg \mathrm{a}$ a 2-GP1, IgM aAnV, IgG aPT, LA were higher in patients with SLE than in the control group, and the levels of IgG and $\lg M a C L$, IgM aß2-GP1, IgG and IgM aAnV, LA were higher in patients with RA v.s. the control group. 